\title{
Simulation-based Evaluation of Spectrum Opportunities in UMTS Cellular Networks
}

\author{
Artur Pereira*, Atílio Gameiro* ${ }^{\dagger}$ \\ * Instituto de Telecomunicações, Universidade de Aveiro, 3810 Aveiro, Portugal \\ * ${ }^{\dagger}$ Departamento de Electrónica \& Telecomunicações, Universidade de Aveiro, 3810 Aveiro, Portugal
}

\begin{abstract}
A scenario based on an UMTS TDD opportunistic cellular system that operates over UMTS FDD licensed cellular networks is considered. Therefore we develop a simulation tool that addresses the goal of analysis and assessment of UMTS TDD opportunistic radio system in a coexistence environment with UMTS FDD primary cellular networks. The communication presents the scenario considered, the main features of the tool, discusses and proposes metrics to evaluate the communication opportunities in UMTS FDD primary cellular networks, and presents numerical simulation results. These show that a moderate number of UMTS TDD available frequencies and a reasonable UMTS TDD opportunistic radio transmission power will allow the deployment of UMTS TDD opportunistic radio networks in a coexistence environment with existing licensed systems.
\end{abstract}

\section{INTRODUCTION}

With the increasing interest of consumers in new wireless applications, demand for the radio spectrum is expected to grow dramatically in the coming years. Today, access to radio spectrum is restricted by an old radio regulatory regime of command and control that emerged over the last 100 years. Large parts of our radio spectrum are allocated to licensed radio services and only a small fraction of radio spectrum is open for free, unlicensed operation. Spectrum utilization studies have shown that most of the assigned spectrum is under-utilized [1], that considerable spectrum is available when both the dimensions of space and time are considered. Hence the problem is in most cases a problem of inefficient spectrum management rather than spectrum shortage. The traditional methods of command and control in spectrum management are no longer optimal, and thus, an alternate remedy to spectrum scarcity is to allow other systems to access such underutilized licensed bands dynamically [2].

Unlicensed devices designed for efficiently using shared spectrum with no or minimal interference to legacy systems are generally referred to as "cognitive radios" [3]. In a broad sense, a cognitive radio is a radio that can change its transmitter parameters based on interaction with the environment in which it operates. In this communication we consider however a narrower definition where the environment awareness considered is restricted to spectrum awareness and term it as opportunistic radios (OR).

In this communication we consider a scenario, where in addition to releasing some new spectrum bands, the regulator allows a secondary market usage, that is secondary users can use the licensed spectrum provided that they do not cause harmful interference to the owners of the licensed band i.e. the primary users [4]. This case study fits with the functional scenario "detection of fading opportunities", that is, the opportunities are generated by the propagation losses conditions and shadowing between the FDD primary users and the TDD opportunistic radio terminal. In such scenario the opportunistic radios autonomously coordinate the usage of shared spectrum. They actively search the radio spectrum for available frequency bands, and use them dynamically adjusting their transmissions to avoid harmful interference with licensed Primary Users (PU). The specific scenario we consider here is the case where an UMTS TDD user can opportunistically use (if no resources are available in the TDD band) the spectrum of FDD systems. For this scenario evaluation and independently of the specific algorithms or protocols used, resorting to simulations is indispensable for the opportunities evaluation of the OR network and its impact on the licensed one. Therefore we develop a simulation tool that allows assessing the coexistence of UMTS TDD OR as a secondary system together with primary UMTS FDD cellular systems.

The simulation platform is based on a system level simulator, designed for the simulation of packet based for UMTS FDD mobile communication systems with extensions to support opportunistic radio systems. The system level simulator considers that there are $N$ cellular systems in a geographical area operating at frequency bands $f_{1}, \cdots, f_{N}$, and a network of ORs which can operate on any of these frequency bands, but whose communication is a second priority one, i.e. the TDD OR can only transmit in the band $f_{n}$ if guaranteed that it does not degrade the performance of the FDD cellular licensed users. For each frequency $f_{n}$ the system level simulator computes, during a duration call, the interference that would be caused by a TDD OR on each user of the cellular network. These values are then used to compute simple statistics for the OR communication opportunities evaluation, in function of the OR transmission power and number of available frequencies

The remainder of this paper is organized as follows. In section 2 , the scenario considered for the development of the simulation tool is described. In section 3 we describe the main features of the simulation tool and the metrics for the spectrum opportunities evaluation and then in section 4 we give some numerical results, and finally the main conclusions of this paper are given in section 5 .

\section{Cellular Scenario Description}

We consider a scenario where the primary cellular network is an UMTS FDD system and the secondary network, a 
centralized cellular network based on the UMTS TDD standard with extra sensing features and able to switch its carrier frequency to UMTS FDD frequencies. This is motivated by the fact that $3 \mathrm{G}$ radio frequency spectrum is an expensive commodity in many countries, and therefore the opportunistic use of these bands could be one way for the owners of the licenses to make extra revenues.

The UMTS FDD use paired uplink and downlink bands and is intended for high mobility applications in macro cell environments with data rates of up to $384 \mathrm{Kbit} / \mathrm{s}$ and because of the paired bands is suited for symmetric traffic. So, an OR system based on UMTS TDD could take advantage of eventual opportunities on these bands and increase the overall UMTS system capacity.

The figure 1 illustrates the concept proposed in this work. Notice that there is an UMTS FDD DL channel with half load occupancy, the UMTS TDD OR detects this opportunity and accommodates an extra capacity.

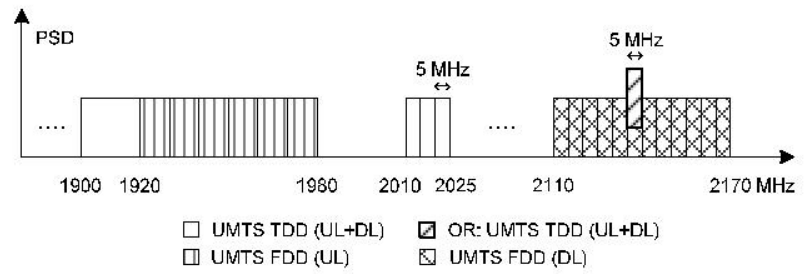

Fig. 1. Using an UMTS FDD UL frequency band in an opportunistic way

We define a PU as a subscriber of the system that legally owns the UMTS FDD frequency band. FDD PUs are not OR aware, i.e. there are no means to exchange information between FDD primary and TDD secondary users provided by a primary system. Specifically, FDD PUs do not provide special signalling in order to access their frequency band. On the other hand the TDD OR is an entity that is able to use the spectrum of licensed users, provided the measurements or information available about this spectrum usage guarantees it will not degrade the quality of PUs communication.

TDD ORs have constrained access to a PU frequency band. A TDD OR can only transmit in the frequency $f_{i}$ if guaranteed that it does not degrade the performance of the UMTS FDD cellular licensed users, i.e. if for a reliable operation $\mathrm{PU}(i)$ requires $\mathrm{SIR}>\gamma(i)$, then it must be guaranteed that the extra interference caused by a TDD user operating in the opportunistic mode does not lead to a violation of this condition.

It is clear from the previous discussion that if a TDD terminal needs to operate in the OR mode (because no resources are available in the licensed TDD band) it needs to accurately compute the interference it may cause on any of the UMTS FDD PUs. The acquisition of this information which can be done through collaborative sensing or eventually through a third party is beyond the scope of this communication. We consider that it is available to the $\mathrm{OR}$ and concentrate on the statistics concerning the opportunity communication available to an $\mathrm{OR}$ assuming such a information available.

The scenario considered in this work is illustrated in figure 2, where there are $N$ cellular systems overlapped in a geographical area and operating at frequency bands $f_{l}, \ldots, f_{N}$. The operation of the TDD OR terminals is explained as follows. If the TDD OR terminal has data to transmit/receive, the base station check if radio resources are available within the TDD licensed band.

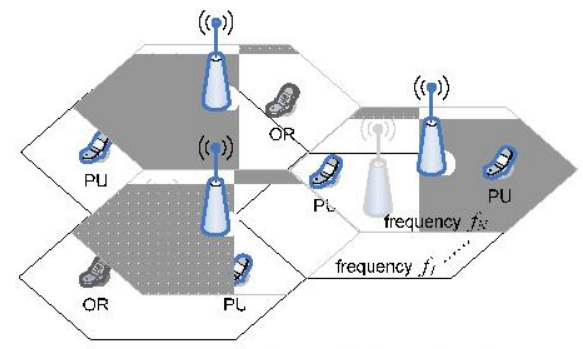

Fig. 2. Multisystem cellular networks

If not, it searches the radio spectrum for available FDD frequency bands, and use them dynamically without decreasing the SIR at FDD PUs locations below a target value $(\gamma)$.

Let us consider PU unit $i$, whose target is a SIR $\geq \chi(i)$. We consider that the attenuation between the FDD BS and FDD PU $i$ is $L(i)$, then in the absence of TDD ORs the FDD PU SIR is given by,

$$
\operatorname{SIR}(i)=\frac{P(i) \times L(i)}{I(i)+N_{o}}
$$

where $N_{o}$ is the noise power and $I(i)$ the cellular interference (intracell and inter-cell).

A TDD $\mathrm{OR}(k)$ located at position so that there is an attenuation $L_{o}(k, i)$ between itself and the FDD PU(i). $P_{o}(i)$ will contribute to a noise level of $P_{o}(k) L_{o}(k, i)$ at FDD PU $i$. Then the FDD PU SIR condition gets,

$$
\frac{P(i) \times L(i)}{I(i)+N+P_{o}(k) \times L_{o}(k, i)}>\gamma(i)
$$

which mean that TDD OR $(k)$ is only allowed to transmit if,

$$
\frac{P(i) \times L(i)}{\gamma(i)}>I(i)+N+P_{o}(k) \times L_{o}(k, i)
$$

for all mobiles $i$ active at a given time.

As the shared spectrum is accessed in a non exclusive way, the maximum allowable TDD OR transmission power depends on propagation losses conditions and shadowing between the TDD OR and the FDD PU terminal. However, in this work, we assume that the power transmitted by the TDD OR is constant, simplifying, in that way, the implementation, but in the other hand increasing the number of transitions (hops) between available frequencies.

\section{SimUlation TOOL DESCRIPTION}

In this section we give an overview of the system level simulation structure, channel models and metrics for the assessment of spectrum opportunities.

\section{A. Structure}

The structure of the system level simulator is shown in figure 3 . The system level simulator interfaces with the link level simulator through look up tables (LUTs) and therefore can be used with a 
large number of different PHY layers, provided the appropriate LUTs are present as input to the simulator. Several propagation and traffic models are available, and the simulator computes the entire channel losses (fast and slow fading), thereby ensuring accuracy in the system level parameters computed. The outputs are the parameters that usually characterize packet transmissions: BER, FER, packet loss, packet delay, OR metrics, etc, and global system and individual statistics can be calculated using these output parameters.

The current simulation platform packet system is based on the High-Speed Downlink Packet Access (HSDPA) MAC layer mechanisms.

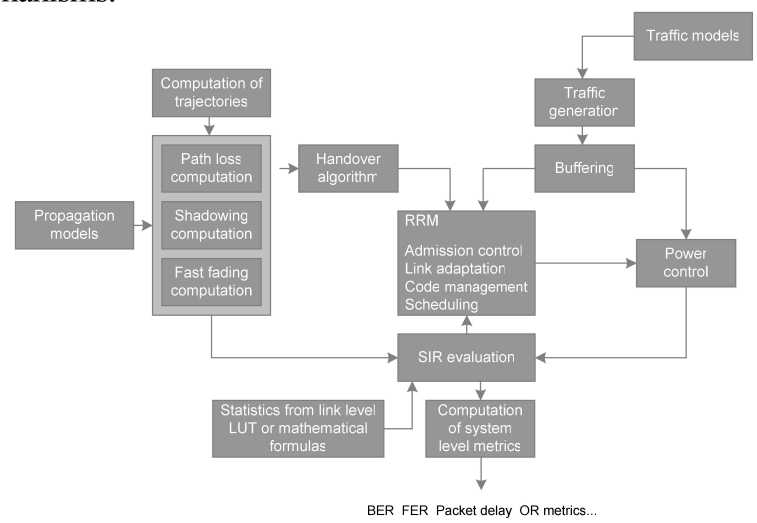

Fig. 3. Structure of system level simulator

At the beginning of each run, mobile stations are created and remain active for the all run duration. These users are randomly uniformly distributed over a hexagonal network of omni directional cells. For each couple of FDD PU and BS, a random shadowing value is drawn, the fast fading is generated and the path loss calculated. Positions, shadowing, path loss and fast fading values are calculated for each time transmission interval, i.e. the FDD PUs are moving. Notices that if an UMTS FDD PU leaves the system, i.e. exceed the cell limit, a new FDD PU is inserted in a random position. Therefore, the number of PUs present in the system is always $K$. In that case, even if a TDD OR is transmitting in the frequency $f_{i}$, the new FDD PU inserted in the cell assumes that the frequency is available and starts its transmission without informing the TDD OR (the FDD PUs and the TDD OR do not exchange information).

\section{B. Channel Models}

The fast fading is generated using Jakes model for fast generation of independent Rayleigh faders [5]. Bi-dimensional shadowing is generated at the beginning of each run. The shadowing is log-normal. The shadowing $S H(x, y, j)$ in $\mathrm{dB}$ between one $\operatorname{MS}(x, y)$ and one $\mathrm{BS} j$ is the sum of two variables,

$$
S H(x, y, f)=\sqrt{0.5} \times\left(F_{0}(x, y)+F_{j}(x, y)\right)
$$

where $F_{0}$, and $F_{j}$ are spatial functions generated using the method described in [6], fulfilling the requirements listed hereafter. $F_{0}$, and $F_{j}$ have a Gaussian distribution, with mean equal to $\sigma$ (the shadowing standard deviation in $\mathrm{dB}$ ), and a spatial correlation given by,

$$
R(d)=e^{-\ln (2) \times \frac{d}{D}}
$$

where $d$ is the distance between two points on the network, and $D$ is the shadowing de-correlation length.

The path loss is expressed with the COST231-Walfish-Ikegami model [7] arguments taking into account base station and receiver heights. The expression of the path loss $L_{b}$ in $\mathrm{dB}$ is given by,

$$
L_{b}=73.24+\left(24.5-\frac{1.5 f_{c}}{925}\right) \log _{10} f_{c}+38 \log _{10} d(6)
$$

where $f_{c}$ denote the carrier frequency in $\mathrm{MHz}$, and $d$ is the distance between two points on the network given in $\mathrm{km}$.

Notice that the platform selects the right speed, carrier frequency, path loss, shadowing and fast fading channel parameters according to the environment chosen for the simulation.

\section{Metrics}

In order to share the spectrum between primary and secondary users, the simulator distributes $K$ FDD PUs in each of the $N$ available frequencies and introduces an OR in that given coverage area. At the beginning, the OR tries to start communicating on frequency $f_{1}$. If the condition $\operatorname{SIR} \geq \gamma(i)$ is satisfied for $i=1 \ldots K$, the OR transmits in that frequency. Otherwise, the OR tries to communicate on frequency $f_{2}, f_{3}$, and so on. This frequency search stops when the OR finds a communication/spectrum opportunity or when no more frequencies are available.

In this context, we have defined the probability of transition, which is the probability of a TDD OR to change its operating frequency during the OR call. If $P_{i j}$ represents the probability of transition, then

$$
P_{i j}=\frac{N t_{i j}}{\sum_{j=0}^{N} N t_{i j}}
$$

where $N t_{i j}$ denote the number of transitions between state $i$ and state $j$ during the OR call.

This metric allow us to define transition diagrams, and then assuming that the system can be described as a Markov state diagram, as shown in figure 4 . We can completely describe the system behaviour using such diagram.

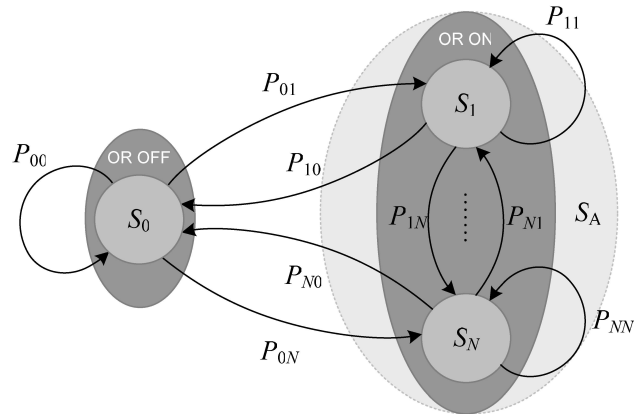

Fig. 4. Markov diagram for the TDD OR carrier frequency transition

Considering $N$ frequencies for the TDD OR communication and assuming no radio resources available in the UMTS TDD network we have $N+1$ states, 
- $S_{0}$ : No opportunities are available in the FDD systems and the TDD OR terminal is OFF.

- $S_{i}$ : The TDD communicates through an FDD band $f_{i}$.

- $P_{i j}$ : Probability of transition from frequency $f_{i}$ to frequency $f_{j}$.

With such a characterization the statistics of the opportunities can be calculated using the transition diagram. Let $\pi_{i} \equiv$ probability that terminal is in state $S_{i}$ then we have,

$$
\vec{\Pi}=\left(\begin{array}{c}
\pi_{0} \\
\vdots \\
\pi_{N}
\end{array}\right)=\overrightarrow{\mathrm{P}} \vec{\Pi}
$$

where $\overrightarrow{\mathrm{P}}$ is the transition probability, i.e. the element $i j$ of $\overrightarrow{\mathrm{P}}$ is $P_{i j}$ [8].

Thus, we can defined the communication probability, obtained as

$$
P_{c}=1-\pi_{0}=\sum_{i=1}^{N} \pi_{i} .
$$

Other statistics of interest are the time that the system spends in $S_{0}$, because no opportunities are available, and the average duration of opportunities. However even when there are opportunities the system may have to switch from frequencies and another statistics of interest is the time between these frequency transitions. To derive such statistics we consider the diagram shown in figure 5 , where $\mathrm{D}$ variable represents an iteration (i.e.: $T_{t i}$ ).

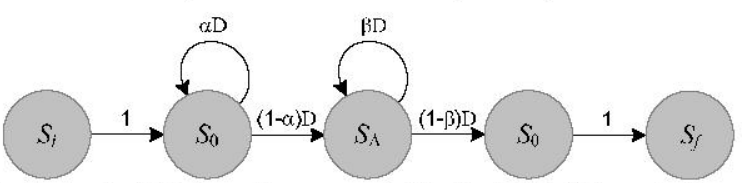

Fig. 5. Equivalent Markov diagram model for the TDD OR carrier frequency transition

We are interested in events of the type such that the system starts as $S_{0}$ and then returns to $S_{0}$ and compute the time spent in the different states. For that and similarly to the procedure done with convolutional codes to characterize the distance profile, we consider the initial and final states to be $S_{0}$. In the diagram we consider $S_{\mathrm{A}}=S_{1} \cup S_{2}$ (only two available frequencies). The use of convolutional theory of Markov diagrams allows us to compute the average number of iterations until the system returns back to $S_{0}$ as,

$$
N_{00}=1+\underbrace{\frac{\alpha}{1-\alpha}}_{\substack{\text { nb of transitions } \\ \text { spent in } S_{0}}}+\underbrace{\frac{\beta}{1-\beta}}_{\substack{\text { nb of transitions } \\ \text { spent in } S_{\mathrm{A}}}}
$$

where $\alpha$ and $\beta$ represents to the probability of transition from $S_{0}$ to $S_{0}$ and $S_{\mathrm{A}}$ to $S_{\mathrm{A}}$, respectively. Therefore the average time spent in $S_{0}$ before entering $S_{\mathrm{A}}$ is

$$
T\left(S_{0}\right)=\frac{\alpha}{1-\alpha} T_{t i}
$$

and the average time spent in $\mathrm{S}_{\mathrm{A}}$ (i.e. with opportunities to communicate) is

$$
T\left(S_{\mathrm{A}}\right)=\frac{\beta}{1-\beta} T_{t i}
$$

Decomposing state $\mathrm{S}_{\mathrm{A}}$ as $S_{1} \cup S_{2}$, as shown in figure 6,

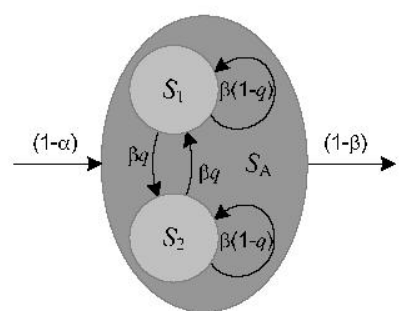

Fig. 6. State $S_{\mathrm{A}}$ of the equivalent Markov diagram model for the TDD OR carrier frequency transition

it is possible to show that during the time $T\left(S_{\mathrm{A}}\right)$ the number of transitions between frequencies $f_{1}$ and $f_{2}$, is given by

$$
N t_{\text {int }}=\frac{\beta q}{1-\beta},
$$

Thus, the average time between frequency switchis is

$$
\Delta t_{S_{1}, S_{2}}=\frac{T\left(S_{\mathrm{A}}\right)}{N t_{\mathrm{int}}}=\frac{\beta T_{t i}}{1-\beta} \frac{1-\beta}{\beta q}=\frac{T_{t i}}{q} .
$$

\begin{tabular}{|c|c|}
\hline Parameter Name & Value \\
\hline \multicolumn{2}{|l|}{ UMTS FDD system } \\
\hline Time transmission interval $\left(T_{t i}\right)$ & $2 \mathrm{~ms}$ \\
\hline Cell type & Omni \\
\hline Cell radius & $577 \mathrm{~m}$ \\
\hline Data rate $\left(R_{b}\right)$ & $12.2 \mathrm{kbps}$ \\
\hline$E_{b} / N_{o}$ target & $9 \mathrm{~dB}$ \\
\hline SIR target $(\gamma)$ & $-16 \mathrm{~dB}$ \\
\hline Spreading factor & 16 \\
\hline Channel Model & Urban \\
\hline Carrier frequency & $2 \mathrm{GHz}$ \\
\hline Channel model & ITU vehicular A \\
\hline Mobile terminals velocity & $30 \mathrm{~km} / \mathrm{h}$ \\
\hline \multicolumn{2}{|l|}{ FDD Primary User (PU) } \\
\hline $\begin{array}{l}\text { Number of primary user(s) per } \\
\text { cell/frequency }(K)\end{array}$ & 5 \\
\hline Sensibility/Power received & $-117 \mathrm{dBm}[9]$ \\
\hline \multicolumn{2}{|l|}{ TDD Opportunistic Radio (OR) } \\
\hline $\begin{array}{l}\text { Number of opportunistic radio(s) } \\
\text { in the cell coverage area }(M)\end{array}$ & 1 \\
\hline $\begin{array}{l}\text { Maximum/Minimum } \\
\text { transmitted }\left(P_{o[\text { maximin }]}\right)\end{array}$ & $10 /-44 \mathrm{dBm}[10]$ \\
\hline Duration call $\left(T_{c d}\right)$ & $90 \mathrm{~s}\left(45000 T_{t i}\right)$ \\
\hline
\end{tabular}

The switching turn-on turn-off times are considered to be instantaneous.

\section{NumERICAL RESUlts}

Table 1. Main parameters used for the simulations

Simulations were carried out to compute the statistics defined in the previous section, related to the $\mathrm{OR}$ communication opportunities. We consider for the reported simulations that the 
information necessary to SIR calculation is perfect, and we do not deal directly with the algorithms to acquire them, since our aim is to analyse the existing opportunities. The main parameters used for the simulations are summarized in table 1. We furthermore consider load characteristics identical in every UMTS FDD cellular system, as referred above, and the frequencies are close enough so that the same statistical models apply.

In order to analyse the impact of the available licensed frequencies on an TDD OR duration call, simulations have been performed considering differents $P_{o}$, a TDD OR duration call equal to 90 seconds and a FDD and TDD terminals velocity equal to 30 $\mathrm{km} / \mathrm{h}$.

The table 2 contains the results related to the probability of each carrier frequency transition, considering $N=2$ and $P_{0}=-30$, $-20,-10,0$ and $10 \mathrm{dBm}$.

Table 2. Probability for each possible carrier frequency transition, considering $N=2$ and $P_{0}=[-30 ; 10] \mathrm{dBm}$

\begin{tabular}{c|ccccc}
\hline \hline $\begin{array}{c}P_{o}(\mathrm{dBm}) \\
\text { Transition }\end{array}$ & -30 & -20 & -10 & 0 & 10 \\
\hline \hline$\hat{P}_{00}$ & 0 & 0.2864 & 0.5011 & 0.5285 & 0.8069 \\
$\hat{P}_{01}$ & 0 & 0.3166 & 0.2396 & 0.2287 & 0.1008 \\
$\hat{P}_{02}$ & 0 & 0.3970 & 0.2593 & 0.2428 & 0.0923 \\
$\hat{P}_{11}$ & 0.9998 & 0.9796 & 0.9348 & 0.6844 & 0.4454 \\
$\hat{P}_{10}$ & 0 & 0.0130 & 0.0242 & 0.2252 & 0.4460 \\
$\hat{P}_{12}$ & 0.0002 & 0.0074 & 0.0410 & 0.0904 & 0.1087 \\
$\hat{P}_{22}$ & 0.9998 & 0.9771 & 0.9402 & 0.7006 & 0.4191 \\
$\hat{P}_{20}$ & 0 & 0.0157 & 0.0234 & 0.2173 & 0.4589 \\
$\hat{P}_{21}$ & 0.0002 & 0.0072 & 0.0364 & 0.0821 & 0.1220 \\
\hline
\end{tabular}

Table 2 shows that the probability of continuing in state $S_{1}$ or $S_{2}$ is low for high values of $P_{0}$; since there are few communication opportunities in the available frequencies, the TDD OR need to frequently change its carrier frequency.

Based on the transitions probabilities (table 2), we can solve expression (8) for $N=2$,

$$
\left[\begin{array}{lll}
P_{00} & P_{01} & P_{02} \\
P_{10} & P_{11} & P_{12} \\
P_{20} & P_{21} & P_{22}
\end{array}\right]\left[\begin{array}{l}
\pi_{0} \\
\pi_{1} \\
\pi_{2}
\end{array}\right]=\left[\begin{array}{l}
\pi_{0} \\
\pi_{1} \\
\pi_{2}
\end{array}\right]
$$

and through (9), determine the probability of communication $P_{c}$. Therefore, for $P_{o}=-10$ and $10 \mathrm{dBm}$ the probability of communication is equal to 95.45 and $29.93 \%$, respectively; we show that the lower the power transmitted by the TDD OR the higher the probability of a TDD OR being able to communicate

The table 3 shows the results related to the average time $T\left(S_{\mathrm{A}}\right)$ and $\Delta t_{S_{1}, S_{2}}$. According to Markov diagram $\alpha=P_{00}, \beta(1-$ $q)=P_{11}=P_{22}$ and $\beta=P_{11}+P_{12}=P_{22}+P_{21}$. Since in practice, because of finite simulation times, the measurement given $\hat{P}_{11} \neq \hat{P}_{22}$, we use $P_{11}=P_{22}=\left(\hat{P}_{11}+\hat{P}_{22}\right) / 2$ and $P_{12}=P_{21}=\left(\hat{P}_{12}+\hat{P}_{21}\right) / 2$. Table 3 shows that the average communication time $T\left(S_{\mathrm{A}}\right)$ decreases with the increase of power transmitted by the $\mathrm{OR}$. To achieve an average communication time equal to the duration call, the TDD OR must transmit at most $-30 \mathrm{dBm}$. In this case, the transitions between available frequencies occur every 10 seconds.

Table 3. Average time in state $S_{\mathrm{A}}$ and between $S_{1}$ and $S_{2}$, considering

\begin{tabular}{c|cc}
\multicolumn{4}{c}{$P_{0}=[-30 ; 10] \mathrm{dBm}$} \\
\hline Av. time & & \\
$P_{0}(\mathrm{dBm})$ & $T\left(S_{\mathrm{A}}\right)$ & $\Delta t_{S_{1}, S_{2}}$ \\
\hline-30 & $90 \times 10^{3}$ & $10 \times 10^{3}$ \\
-20 & 137,3728 & 270,0411 \\
-10 & 82,0336 & 50,4496 \\
0 & 7,0395 & 18,0580 \\
10 & 2,4204 & 9,4937 \\
\hline
\end{tabular}

\section{CONCLUSIONS}

In this paper, we have considered the challenge of spectrumsharing between licensed cellular mobile communications system and virtually unlicensed users that share the licensed spectrum but have a lower priority and are not allowed to degrade the performance of the PUs. A realistic evaluation of the coexistence of such systems can only be done resorting to simulations, and therefore a system level simulator, where $N$ cellular systems operating at different frequencies allow secondary UMTS TDD OR users to share their spectrum was developed. In a general way, simulation results have shown, considering realistic propagation environments, that a moderate number of available frequencies and a reasonable TDD OR transmission power will allow the deployment of UMTS TDD OR networks in a coexistence environment with existing licensed systems.

\section{REFERENCES}

[1] Shared Spectrum Company, "New York City Spectrum Occupancy Measurements September 2004".

[2] A. Ghasemi and E. S. Sousa, "Collaborative Spectrum Sensing for Opportunistic Access in Fading Environments," New Frontiers in Dynamic Spectrum Access Networks, First IEEE International Symposium, page 131-136, November 2005.

[3] J. Mitola, III, "Cognitive Radio for Flexible Mobile Multimedia Communications," Mobile Multimedia Communications, IEEE International Workshop, page 3, 1999.

[4] R. Brodersen et al, "CORVUS: A Cognitive Radio Approach for Use of Virtual Unlicensed Spectrum", University of Berkeley.

[5] Y. Li and X. Huang, "The Generation of Independent Rayleigh Faders" Proceedings of IEEE, ICC" $00,2000$.

[6] X. Cai, Member IEEE, and G. B. Giannakis, Fellow IEEE, "A twoDimensional Channel Simulation Model for Shadowing Processes", IEEE Transactions On Vehicular Technology, vol. 52, November 2003.

[7] COST 231Final Report. Available: $h$ ttp://www.lx.it.pt/cost231/

[8] Ross, Sheldon M. (1997), Introduction to Probability Models, 6th edition, Berkeley, CA: Academic Press

[9] 3GPP, "Technical Specification Group Radio Access Network; User Equipment (UE) radio transmission and reception (FDD) (Release 6)," TS 25.101. v6.13.0, October 2006

[10] 3GPP, "Technical Specification Group Radio Access Network; User Equipment (UE) radio transmission and reception (TDD) (Release 6)," TS 25.102. v6.5.0, October 2006. 